\title{
Influência do hiperparatireoidismo secundário grave no estado nutricional de pacientes com insuficiência renal crônica
}

\author{
Influence of severe secondary hyperparathyroidism in the \\ nutritional status of patients with chronic renal failure
}

Bárbara Santarosa Emo PETERS'

Vanda JORGETTI2

Lígia Araújo MARTINI ${ }^{3}$

\section{RE S U M O}

\section{Objetivo}

Avaliar o efeito do paratormônio na ingestão alimentar e sua relação com a composição corporal de pacientes em programa de hemodiálise com hiperparatireoidismo secundário.

\section{Métodos}

Foram avaliados 26 pacientes adultos, com média de idade de $47 \pm 8$ anos, divididos em dois grupos, de acordo com os níveis de paratormônio, e pareados por sexo. No grupo $1(n=3)$ os níveis de paratormônio eram maiores que $300 \mathrm{pg} / \mathrm{ml}(1486 \pm 920 \mathrm{pg} / \mathrm{ml})$, e no grupo 2 ( $\mathrm{n}=13)$, menores que $300 \mathrm{pg} / \mathrm{ml}(199,8 \pm 122,2 \mathrm{pg} / \mathrm{ml})$. Todos os pacientes realizaram registro alimentar de três dias, medidas antropométricas e exames bioquímicos séricos para avaliar o metabolismo ósseo.

\section{Resultados}

Os pacientes do grupo 1 apresentaram índice de massa corporal, porcentagem da circunferência muscular do braço, da prega cutânea triciptal e de gordura corpórea, significativamente menores que os pacientes do grupo $2(p<0,05)$. Houve correlação negativa e significante entre o paratormônio e a gordura corporal $(r=-0,6$; $p<0,05)$. Em adição, a análise de regressão linear múltipla mostrou que o paratormônio, junto com o tempo em diálise, explicam em $42 \%$ a diminuição da gordura corporal total. Quanto à ingestão de nutrientes e aos marcadores bioquímicos, com exceção do paratormônio, não houve diferença estatisticamente significante entre os dois grupos. As ingestões de energia, proteína e cálcio estavam abaixo das recomendações para pacientes em diálise, e a ingestão de fósforo foi em média $11 \pm 6 \mathrm{mg} / \mathrm{kg} / \mathrm{dia}$ e $15 \pm 5 \mathrm{mg} / \mathrm{kg} / \mathrm{dia}$, e considerada adequada para esta população.

\footnotetext{
1 Mestranda em Saúde Pública, Departamento de Nutrição, Faculdade de Saúde Pública, Universidade de São Paulo. São Paulo, SP, Brasil.

2 Disciplina de Nefrologia, Faculdade de Medicina, Universidade de São Paulo. São Paulo, SP, Brasil.

3 Departamento de Nutrição, Faculdade de Saúde Pública, Universidade de São Paulo. Av. Dr. Arnaldo, 715, Cerqueira César, 01246-904, São Paulo, SP, Brasil. Correspondência para/Correspondence to: L.A. MARTINI.
} 


\section{Conclusão}

Com ingestão alimentar semelhante, quanto maior o nível de paratormônio e o tempo em diálise, menor é a gordura corporal nos pacientes com hiperparatireoidismo secundário grave, o que mostra que níveis altos de paratormônio contribuem para alterações no estado nutricional nestes pacientes.

Termos de indexação: avaliação nutricional; diálise renal; hiperparatireoidismo; pacientes.

\section{A B S T R A C T}

\section{Objective}

To evaluate parathyroid hormone effects in the nutritional status of hemodialysis patients with secondary hyperparathyroidism.

\section{Methods}

Twenty-six adult patients with a mean age of $47 \pm 8$ years were evaluated. The patients were divided into two groups according to their levels of parathyroid hormone and matched for gender. Patients in group $1(n=13)$ presented parathyroid hormone levels above 300pg/ml (1486 $\pm 920 \mathrm{pg} / \mathrm{ml})$ and patients in group 2 ( $n=13)$ below 300pg/ml (199.8 $\pm 122.2 \mathrm{pg} / \mathrm{ml})$. The following parameters were also assessed: 3-day food diaries, anthropometric data and biochemical markers of bone metabolism.

\section{Results}

Group 1 presented significantly lower body mass index, midarm muscle circumference, skinfold thickness and body fat compared with patients from group $2(p<0.05)$. There was a negative and significant correlation between parathyroid hormone and total body fat $(r=-0.6 ; p<0.05)$. In addition, in the multiple regression analysis, the parathyroid hormone and the time in hemodialysis explain in $42 \%$ the decrease in total body fat. Concerning nutrient intakes and biochemical markers, except for parathyroid hormone, there was no statistically significant difference between the two groups. All patients presented lower energy, protein and calcium intakes than those recommended for dialysis patients. Phosphorus intake was $11 \pm 6$ and $15 \pm 5 \mathrm{mg} / \mathrm{kg} / \mathrm{day}$ for groups 1 and 2, respectively, values considered adequate for this population.

\section{Conclusion}

In conclusion, elevated levels of parathyroid hormone and time in hemodialysis have a negative effect on total body fat and can influence the nutritional status of chronic renal failure patients.

Indexing terms: nutritional assessment; renal dialysis; hyperparathyroidism; patients.

\section{N T R O D U Ç Ã O}

O hiperparatireoidismo secundário (HPT2) é uma complicação freqüente em pacientes com insuficiência renal crônica (IRC) ${ }^{1-3}$, podendo acometer $5 \%$ a $25 \%$ dos pacientes portadores de IRC, incluindo os que se encontram em programa regular de hemodiálise (HD). Com a manutenção do programa dialítico, aproximadamente $50 \%$ dos pacientes podem desenvolver esta enfermidade ${ }^{4-7}$.

A característica principal dessa enfermidade é a hiperplasia das glândulas paratireóide e aumento da síntese e secreção do paratôrmonio (PTH), que são induzidos pela ocorrência de hipocalcemia, hiperfosfatemia, deficiência de calcitriol $\left[1,25(\mathrm{OH})_{2} \mathrm{D}_{3}\right]$, e da resistência esquelética à ação do PTH ${ }^{4,8}$. Seu quadro clínico é variado em conseqüência de alterações fisiopatológicas ${ }^{9}$, podendo ocorrer dores ósseas e musculares, prurido, calcificação das partes moles, calcifilaxia, fraturas, deformidades ósseas e osteoclastomas, perda de apetite, anormalidade no paladar e perda de peso em intensidade variável $^{3,8,10}$.

Vários trabalhos ${ }^{11-16}$ têm demonstrado a alta prevalência de desnutrição energético - protéica em pacientes com IRC em tratamento dialítico, sendo um importante fator para o aumento da morbidade e mortalidade nestes pacientes. A 
desnutrição pode ocorrer devido a vários fatores, como anorexia, perda de nutrientes e catabolismo durante o processo de diálise, doenças concomitantes, acidose metabólica, intolerância à glicose, aumento do nível de citoquinas, inflamação crônica e alterações hormonais ${ }^{10,12,14}$. Níveis elevados de PTH, como os observados no HPT2, também contribuem nas anormalidades nutricionais encontradas nestes pacientes ${ }^{14}$.

Poucos estudos ${ }^{7,12}$ avaliaram os efeitos do HPT2 na composição corporal desses pacientes. Assim, o objetivo deste trabalho foi avaliar o efeito PTH na ingestão alimentar e sua relação com a composição corporal de pacientes com IRC e HPT2.

\section{MÉ TO D O S}

Este estudo foi realizado com pacientes do Ambulatório de Osteodistrofia Renal da disciplina de Nefrologia da Faculdade de Medicina da Universidade de São Paulo. Participaram deste estudo pacientes com HPT2 e IRC, em programa de hemodiálise. Nesse ambulatório são atendidos pacientes de diversas unidades de diálise da cidade de São Paulo e cidades vizinhas. Foram excluídos pacientes com doenças concomitantes, infecções recentes ( $<3$ meses), tuberculose (TB) em tratamento, doença intestinal, alcoolismo crônico, síndrome da imunodeficiência adquirida (HIV), doenças malignas, insuficiência cardíaca e pulmonar grave e insucessos de transplante renal nos últimos seis meses.

Foram avaliados 26 pacientes adultos com idade de $47 \pm 8$ anos, sendo 9 mulheres e 17 homens. Os pacientes foram divididos em dois grupos, pareados por sexo, de acordo com o nível de PTH. O grupo 1 ( $n=13)$, constituído por pacien-tes com PTH acima de $300 \mathrm{pg} / \mathrm{ml}(1486 \pm 920 \mathrm{pg} / \mathrm{ml})$, e o grupo $2(n=13)$ constituído por pacientes com PTH abaixo de 300pg/ml (199,8 $\pm 122,2 \mathrm{pg} / \mathrm{ml})$. O tempo médio de HD dos pacientes do grupo 1 era de $10 \pm 2,2$ anos e do grupo 2 de $4 \pm 1,4$ anos. Os grupos foram divididos com estes valores de PTH pois, para pacientes com IRC em estágio
5, o K/DOQI de 2003 recomenda que o PTH sérico fique entre 150 e $300 \mathrm{pg} / \mathrm{ml}^{17}$.

Todos os pacientes realizaram registro alimentar de três dias para a avaliação da ingestão alimentar (energia, proteína, cálcio e fósforo), medidas antropométricas (peso, altura, circunferência do braço e pregas cutâneas bicipital, tricipital, abdominal e subescapular), e foram dosados exames bioquímicos séricos (cálcio total, cálcio iônico, fósforo e PTH).

Para a avaliação da ingestão alimentar, foi utilizado o registro alimentar de três dias. O próprio paciente ou o responsável anotaram em um formulário, especialmente desenhado, todos os alimentos e bebidas consumidos ao longo de três dias, sendo dois dias durante a semana - um de hemodiálise e outro não, e um no final de semana.

As medidas antropométricas foram realizadas pelo mesmo examinador, no momento da consulta, no próprio ambulatório e no período interdialítico em todos os pacientes. Foi utilizado o peso seco, relatado pelo paciente. A estatura foi obtida por meio de uma barra metálica com escala em centímetros, acoplada na balança de plataforma. Estes dados foram utilizados para o cálculo do índice de massa corporal (IMC), que relaciona o peso pela altura, em metros ao quadrado. As pregas cutâneas foram obtidas utilizando o adipômetro Lange (Lange Skinfold Caliper) em triplicata, adotando-se o valor médio para a obtenção da porcentagem de gordura corporal, por meio da equação proposta por Durnin $\&$ Wormersley ${ }^{18}$. Para a obtenção da circunferência muscular do braço utilizamos uma fita métrica flexível e inelástica. Estas medidas foram realizadas no braço contrário à fístula arteriovenosa.

Os pontos de corte utilizados para classificação da adequação da PCT e CMB foram os propostos por Blackburn \& Thorton ${ }^{19} \mathrm{e}$, para a porcentagem de gordura corporal, os valores propostos por Lohman ${ }^{20}$.

Para os cálculos dos valores de IMC, porcentagem da Circunferência Muscular do Braço (\%CMB), da Prega Cutânea Triciptal (\%PCT), de 
Gordura Corpórea (\%GC), e a média das quantidades de energia, proteína, cálcio e fósforo consumidas pelo paciente, utilizamos o software Sistema de apoio e Decisão em Nutrição - versão 2.5 do Departamento de Informática em Saúde - Unifesp/ $\mathrm{EPM}^{21}$

As dosagens dos exames bioquímicos foram realizadas no laboratório do Instituto Central do Hospital das Clínicas da Faculdade de Medicina da Universidade de São Paulo (ICHC-FMUSP). A análise do cálcio total empregou o método colorimétrico (valores normais: 8,4 a 10,2mg/dl) do fósforo sérico, o método colorimétrico (valores normais: 2,4 a 4,6mg/dl), e do PTH o método de ensaio imunoradiométrico (valores normais: 6 a $40 \mathrm{pg} / \mathrm{ml})$.

Para a análise estatística, foi utilizado o programa SPSS for Windows, versão 11.0 (SPSS, Inc., Chicago, IL, USA). Os resultados são apresentados em média e desvio padrão. As variações encontradas foram consideradas significativas se $p<0,05$. Para a verificação da distribuição das variáveis estudadas, utilizou-se o teste de Kolmogorov-Smirnov, e quando consideradas com distribuição normal, foram aplicados o teste " $\mathrm{t}$ " de Student e o Coeficiente de Correlacão de Pearson. Foi realizada também a análise de regressão linear múltipla, considerando como variável dependente a porcentagem de gordura corporal. O PTH e o tempo em diálise como variáveis independentes.

\section{RE S U L T A D O S}

Na Tabela 1 estão as características gerais, a ingestão alimentar e os parâmetros bioquímicos dos pacientes participantes do estudo. Não há diferença com relação à idade média entre os grupos, porém os pacientes do grupo 1 estavam em diálise há mais tempo $(10,0 \pm 2,2$ vs 4,3 $\pm 1,4$ anos) e apresentavam menor IMC. Com relação à etiologia da doença renal, não existiam informações completas de todos os pacientes, porém a doença de base que poderia apresentar alguma alteração no estado nutricional seria o diabetes. Este foi encontrado em três pacientes do grupo 2, e nenhum paciente do grupo 1.

Comparando os dois grupos quanto à ingestão alimentar, não houve diferenças significantes (Figura 1). Entretanto a porcentagem de adequação da ingestão alimentar, em relação às recomendações pelo National Kidney Foundation - Disease Outcome Quality (NKF-DOQ), revelou que as ingestões de energia (grupo 1: $22,8 \pm 11,7 \mathrm{kcal} / \mathrm{kg} / \mathrm{dia}$ vs. grupo $2: 31,8 \pm 11,1 \mathrm{kcal} / \mathrm{kg} / \mathrm{dia})$, proteína $(0,9 \pm 0,5 \mathrm{~g} / \mathrm{kg} / \mathrm{dia}$ vs $1,2 \pm 0,4 \mathrm{~g} / \mathrm{kg} / \mathrm{dia})$ e cálcio $(358,3 \pm 176,2 \mathrm{mg} / \mathrm{dia}$ vs $471,4 \pm 241,1 \mathrm{mg} / \mathrm{dia})$

Tabela 1. Características gerais, ingestão alimentar e parâmetros bioquímicos dos pacientes com insuficiência renal crônica.

\begin{tabular}{|c|c|c|c|c|c|c|c|}
\hline \multirow[b]{2}{*}{ Tempo em HD } & \multicolumn{3}{|c|}{ Grupo 1} & \multicolumn{3}{|c|}{ Grupo 2} & \multirow{2}{*}{$\frac{p}{0,001}$} \\
\hline & 10,0 & \pm & $2,2^{a}$ & 4,3 & \pm & 1,4 & \\
\hline Idade (anos) & 44,3 & \pm & 10,4 & 50,4 & \pm & 6,9 & 0,074 \\
\hline Peso $(\mathrm{kg})$ & 58,2 & \pm & 10,8 & 68,6 & \pm & 18,3 & 0,090 \\
\hline Pacientes diabéticos & 0 & \pm & 0 & 3,0 & \pm & 0 & \\
\hline IMC & 21,5 & \pm & 3,2 & 25,5 & \pm & 5,8 & 0,037 \\
\hline \multicolumn{8}{|l|}{ Ingestões } \\
\hline Energia $(\mathrm{kcal} / \mathrm{kg} / \mathrm{d})$ & 22,8 & \pm & 11,7 & 31,8 & \pm & 11,1 & 0,058 \\
\hline Proteína $(\mathrm{g} / \mathrm{kg} / \mathrm{d})$ & 0,9 & \pm & 0,5 & 1,2 & \pm & 0,4 & 0,175 \\
\hline Cálcio (mg) & 358,3 & \pm & 176,2 & 471,1 & \pm & 241,1 & 0,185 \\
\hline Fósforo (mg/kg/d) & 11,1 & \pm & 6,6 & 15,0 & \pm & 5,2 & 0,112 \\
\hline \multicolumn{8}{|c|}{ Parâmetros Bioquímicos } \\
\hline Cálcio total (mg/dl) & 9,5 & \pm & 1,1 & 10,1 & \pm & 1,0 & 0,580 \\
\hline Cálcio iônico (mg/dl) & 4,9 & \pm & 0,8 & 5,2 & \pm & 0,5 & 0,059 \\
\hline Fósforo sérico (mg/dl) & 6,6 & \pm & 1,5 & 6,0 & \pm & 1,8 & 0,368 \\
\hline PTH (pg/ml) & 486,0 & \pm & 919,9 & 199,8 & \pm & 122,2 & 0,000 \\
\hline
\end{tabular}

a $\bar{X} \pm D P ; P T H=$ paratormônio.

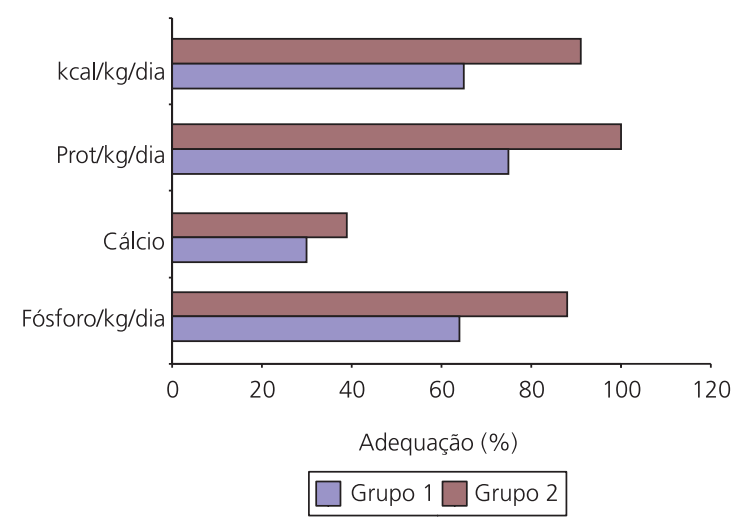

Figura 1. Porcentagem de adequação da ingestão alimentar entre os grupos de pacientes com insuficiência renal crônica, em relação às recomendações da National Kidney Foundation - Disease Outcome Quality. 
estão abaixo das recomendações para pacientes em diálise (energia: $35 \mathrm{kcal} / \mathrm{kg} / \mathrm{dia}$, proteína: 1,2g/kg/dia, cálcio: $1200 \mathrm{mg} /$ dia). Observa-se que a ingestão de cálcio dos alimentos não atingiu $50 \%$ da recomendação em nenhum dos grupos, a ingestão de fósforo foi, em média, $11 \pm 6$ e $15 \pm 5 \mathrm{mg} / \mathrm{kg} / \mathrm{dia}$ (grupos 1 e 2, respectivamente), considerada adequada para esta população (17mg/kg/dia).

Com relação aos exames bioquímicos não houve diferença quanto ao cálcio total $(9,5 \pm 1,1$ vs. $10,1 \pm 1,0)$, cálcio iônico $(4,9 \pm 0,8$ vs. 5,2 $\pm 0,5)$ e fósforo sérico $(6,6 \pm 1,5$ vs. $6,0 \pm 1,8)$ entre os grupos. O PTH foi significantemente mais elevado no grupo $1(1486,0 \pm 919,9$ vs. 199,8 $\pm 122,2)$.

Quanto às medidas antropométricas, avaliando individualmente os pacientes do grupo 1, observou-se predominância de algum grau de desnutrição, tanto em relação à CMB (85\%), quanto em relação à $\mathrm{PCT}(70 \%)$. Ao avaliar a gordura corporal total (GCT), 38\% dos pacientes encontravam-se com reservas diminuídas. Já no grupo 2, houve predominância de eutrofia em relação à CMB (62\%) e à PCT (69\%), e quanto à $\mathrm{GCT}$, houve predominância de obesidade e sobrepeso. Ainda neste grupo, também houve presença de correlação negativa entre a \%CMB e o PTH $(r=-0,62, p<0,05)$.

Na comparação entre os grupos, o grupo 1 apresentou índice de massa corporal (IMC), porcentagem da circunferência muscular do braço (\% CMB), porcentagem da prega cutânea triciptal (\% PCT) e porcentagem de gordura corporal (\% $\mathrm{GC}$ ), significantemente menores que os pacientes do grupo 2 ( $21 \pm 3$ vs. $25 \pm 5 ; 84 \pm 8$ vs. $95 \pm 16$; $83 \pm 39$ vs. $126 \pm 51 ; 21 \pm 9$ vs. $29 \pm 5$, respectivamente) (Figura 2). Além disso, foi observada uma correlação negativa entre o PTH e a porcentagem de gordura corporal ( $\% \mathrm{GC}, \mathrm{r}=-0,54$, $p<0,05)$ (Figura 3) no mesmo grupo.

No modelo de regressão linear múltipla, o PTH e o tempo em diálise em conjunto explicam $42 \%$ da variação da gordura corporal na amostra estudada $\left(R_{\text {ajustado }}=0,416, p=0,009\right)$ (Tabela 2).

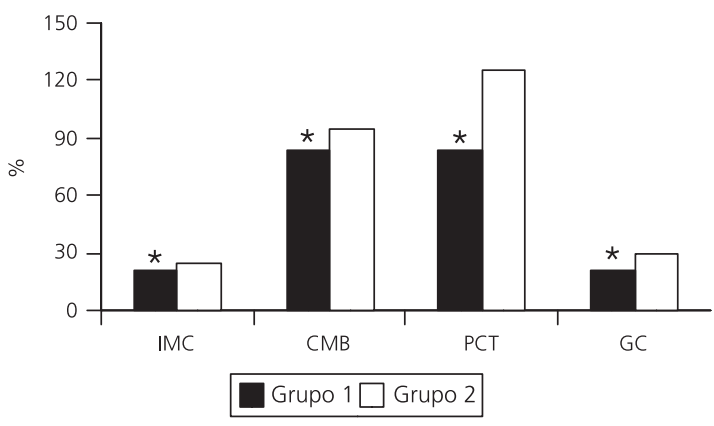

Figura 2. Comparação da composição corporal entre os grupos de pacientes com insuficiência renal crônica.

$\mathrm{IMC}=$ Índice de massa corporal; $\mathrm{CMB}=$ circunferência muscular do braço; PCT= prega cutânea do tríceps; GC= gordura corporal; ${ }^{*} p<0,05$.

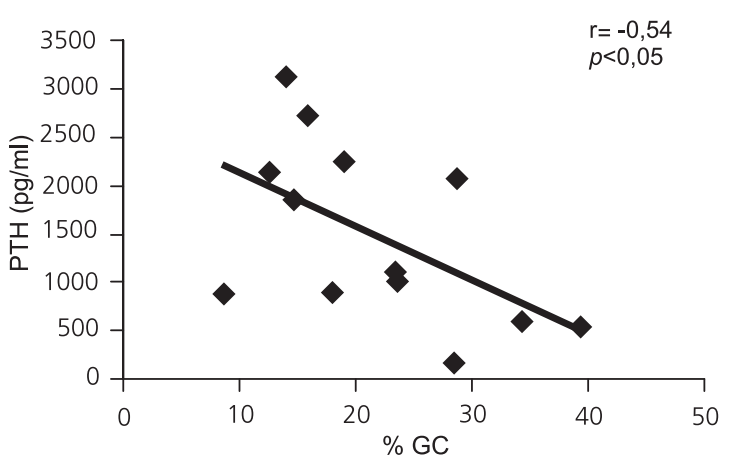

Figura 3. Correlação entre os valores de PTH (pg/ml) e da porcentagem de Gordura Corporal encontrados no Grupo 1.

$\mathrm{PTH}=$ paratormônio. \% GC= porcentagem de gordura corporal.

Tabela 2. Regressão linear múltipla para a porcentagem de gordura corporal (\%GC) dos pacientes com insuficência renal crônica dos grupos 1 e 2 .

\begin{tabular}{lccccc}
\hline $\begin{array}{l}\text { Variável } \\
\text { Dependente }\end{array}$ & $\begin{array}{c}\text { Variáveis } \\
\text { Independentes }\end{array}$ & $\beta$ & $\mathrm{R}^{2}$ & $p$ & $p$ modelo \\
\hline$\% G C$ & PTH & $-0,0056$ & 0,359 & 0,001 & 0,002 \\
& PTH & $-0,0034$ & & 0,068 & \\
$\% G C$ & Tempo HD & $-0,386$ & 0,416 & 0,262 & 0,009 \\
\hline
\end{tabular}

$\mathrm{PTH}=$ paratormônio; Tempo HD= tempo em hemodiálise.

\section{I S C U S S Ã O}

Este trabalho teve como ponto mais relevante a correlação negativa entre PTH e a gordura corporal, refletindo o efeito catabólico que os elevados níveis contínuos de PTH exercem 
nestes pacientes. Além disso, o PTH e o tempo em hemodiálise são responsáveis por $42 \%$ da diminuição da gordura corporal na amostra estudada. Alguns trabalhos ${ }^{22-24}$ têm sugerido que elevados níveis de PTH podem, de forma direta ou indireta, exercer uma ação tóxica sobre o metabolismo energético e principalmente protéico, promovendo catabolismo protéico e balanço nitrogenado negativo. Existem evidências sugerindo que o PTH pode ser uma toxina urêmica, isto porque muitas das manifestações da síndrome urêmica podem ser produzidas pelo excesso de $\mathrm{PTH}^{23-28}$. Alterações no metabolismo lipídico também são observadas com a elevação dos níveis de PTH, podendo levar a alterações no metabolismo muscular, inibindo a produção, transporte e utilização de energia'12,29. Rezende et al. ${ }^{12}$, comparando o estado nutricional de pacientes em HD com e sem HPT2, observaram que o nitrogênio uréico sangüíneo foi significantemente maior e que a taxa de catabolismo protéico foi mais elevada nos pacientes com HPT2, mesmo com semelhante ingestão protéica.

Neste estudo, observou-se que o grupo 1 apresentou valores de IMC, \%CMB, \%PCT e $\%$ GC, significativamente menores que os pacientes do grupo 2. Apesar de trabalhos mostrarem que a somatória das pregas cutâneas, método utilizado para a avaliação da composição corporal neste estudo, é uma técnica simples, não invasiva e de baixo custo, pode ser uma técnica propensa à falta de precisão, devido a alterações na hidratação e conseqüente turgor da pele $\mathrm{1}^{13-16,30,31}$. Entretanto, estudo realizado por Kamimura et al. ${ }^{32}$, comparando três métodos de análise de composição corporal em pacientes em diálise (medidas das pregas cutâneas, impedância bioelétrica e raios infra vermelhos), demonstrou que as duas primeiras eram mais fidedignas que a última, mostrando que a técnica de pregas cutâneas é uma técnica simples, estável e útil para determinar a composição corporal destes pacientes. Esses mesmos autores ${ }^{33}$, comparando as pregas cutâneas, a impedância bioelétrica com o DEXA verificaram que os resultados de gordura corporal obtidos pelos três métodos não apresentaram diferença estatística entre si, mostrando, mais uma vez, que a técnica de pregas cutâneas é valida para a obtenção de dados de composição corporal desses pacientes.

Apesar de, no presente estudo, as medidas antropométricas não terem sido tomadas no momento pós-diálise, estas foram realizadas no momento interdiálitico em todos os pacientes, além disso os pacientes apresentavam-se estáveis, sem febre e não edemaciados. Portanto, desde que estas medidas sejam realizadas com os devidos cuidados, acreditamos que os resultados antropométricos refletem com fidedignidade o estado nutricional destes indivíduos.

O controle do fósforo sérico é muito importante em pacientes com HPT2, pois inibe diretamente a proliferação celular das glândulas paratireoides ${ }^{2,29}$. Uma das formas de manter os valores de fósforo sérico dentro dos limites de normalidade $(2,4$ a 4,6mg/dl) é por intermédio da restrição dietética, o que acaba entrando em conflito com a necessidade diária de ingestão protéica para estes pacientes, que são hipercatabólicos (1,0 a 1,2g/kg/dia) 27,34,38, uma vez que os alimentos ricos em fósforo são também ricos em proteína. Neste trabalho a quantidade de energia, proteína e cálcio ingerida foi similar entre os dois grupos estudados, porém foi menor que as quantidades recomendadas pela National Kidney Foundation - Disease Outcome Quality. A ingestão de cálcio nestes pacientes foi deficiente pois, os alimentos ricos em cálcio, como leite e derivados, também são ricos em fósforo, levando os pacientes a diminuírem a ingestão destes alimentos. Este fato ressalta a importância de uma correta prescrição da utilização de suplementos. A quantidade de fósforo ingerida também foi similar entre os grupos, mas este se manteve dentro das recomendações para pacientes com HPT2, em torno de 500 a $700 \mathrm{mg} / \mathrm{dia}$ ou 11 a $15 \mathrm{mg} / \mathrm{kg} / \mathrm{dia}$. Esta ingestão dietética insuficiente pode também estar contribuindo para o deficiente estado nutricional detectado nesses pacientes, pois a dieta, além de ser hipocalórica, também era hipoprotéica. 


\section{O N C L U S Ã O}

Com a mesma ingestão alimentar, quanto maior o nível de PTH e o tempo em hemodiálise, menor será a gordura corporal nos pacientes com HPT2 grave. Portanto, altos níveis de PTH contribuem para os efeitos deletérios na composição corporal de pacientes renais crônicos e piora do seu estado nutricional.

\section{REFER Ê N CIAS}

1. Slatopolsky E. The role calcium, phosphorus and vitamin D metabolism in the development of secondary hyperparathyroidism. Nephrol Dial Transplant. 1998; 13(3):3-8.

2. Denda M, Finch J, Slatoolsky E. Phoshorus accelerates the develoment of parathyroid hyerplasia and sedondary hyperparathyroidism in rats with renal failure. Am J Kidney Dis. 1996; 28(4):596-602.

3. Llach F, Massry SG. On the mechanism of secondary hyperparathyroidism in moderate renal insufficiency. J Clin Endocrinol Metabol. 1985; 61(4):601-6.

4. Salem MM. Hyperparathyroidism in the hemodialysis population: A survey of 612 patients. Am J Kidney Dis. 1997; 29(6):862-5.

5. Slatopolsky E, Brown A, Dusso A. Role phosphorus in the pathogenesis of secondary hyerparathyroidism. Am J Kidney Dis. 2001; 37(1 Suppl 2):54-7.

6. Kuizon B, Salusky IB. Cell biology of renal osteodystrophy. Pediatr Nephrol. 2002; 17:777-89.

7. Khajehdehi P, Ali M, Al-Gebory F, Henry G, Bastani $B$. The effects of parathyroidectomy on nutrition and biochemical status of hemodialysis patients with severe secondary hyperparathyroidism. J Renal Nutr. 1999; 9(4):186-91.

8. Drueke TB, Zingraff J. The dilemma of parathyroidectomy in chronic renal failure. Curr Opin Nehrol Hypertens. 1994; 3(4):386-95.

9. Sugano LK. Paratireoidectomia total e autotransplante no tratamento do hiperparatireoidismo secundário [dissertação]. São Paulo: Faculdade de Ciências Médicas da Santa Casa de São Paulo, Universidade de São Paulo; 1999.

10. Brown EM, Wilson RE, Eastman RC, Pallotta J, Marynick SP. Abnormal regulation of parathyroid hormone relese by calcium in secondary hyperparathyroidism due to chronic renal failure. J Clin Endocrinol Metabol. 1982; 54(1):172-9.

11. Agudelo MA. Factores que inciden en el estado nutricional en los pacientes en tratamiento de hemodiálisis. Lecturas sobre nutrición. 1999; 6(2):55-7.

12. Rezende LTT, Cuppari L, Carvalho AB, Canziani MEF, Manfredi SR, Cendoroglo $M$, et al. Nutritional status of hemodialysis patients with secondary hyperparathyroydism. Braz J Med Biol Res. 2000; 33(11):1305-11.

13. Woodrow G, Oldroyd B, Smith MA, Turney JH. Measurement of body composition in chronic renal failure: comparison of skinfold anthropometry and bioelectrical impedance with dual energy X-ray absorptiometry. Eur J Clin Nutr. 1996; 50(5):295-301.

14. Johansen LK, Kaysen GA, Young BS, Hung AM, Silva M, Chertow GM. Longitudinal study of nutritional status, body composition, and physical function in hemodialysis patients. Am J Clin Nutr. 2003; 77(4):842-6.

15. Chertow GM, Lowrie EG, Wilmore DW, Gonzalez J, Lew NL, Ling J, et al. Nutritional assessment with bioelectrical impedance analysis in maintenance hemodialysis patients. J Am Soc Nephrol. 1995; 6(1):75-81.

16. Stenver DI, Gotfredsen A, Hilsted J, Nielsen B. Body composition in hemodialysis patients measured by dual - energy X-ray absorptiometry. Am J Nephrol. 1995; 15(2):105-10.

17. National Kidney Foundation. K/DOQI Clinical Practice Guideline for Bone Metabolism and Disease in Chronic Kidney Disease. Am J Kidney Dis. 2003; 42 Suppl 3:S1-S202.

18. Durnin JV, Womersley S. Body fat assessed from total body density and its estimation from skinfold thickness: measurements on 481 men and women age from 16 to 72 years. Br J Nutr. 1974; 32(1): 77-97.

19. Blackburn GL, Thornton PA. Nutritional assessment of the hospitalized patients. Med Clin North Am. 1979; 63(5):11103-15

20. Lohman TG. Advances in body composition assessment. Champaign: Human Kinetics Books; 1992.

21. Anção MS, Cuppari L, Tudisco ES, Draibe SA, Sigulem D. Versão 2.5: sistema de apoio e decisão em nutrição. São Paulo: Centro de Informática em Saúde da Universidade Federal de São Paulo, Escola Paulista de Medicina; 2000.

22. Smogorzeuski M, Piskorska G, Borum PR, Massry SG. Chronic renal failure, parathyroid hormone and fatty acids oxidation in skeletal muscle. Kidney Int. 1988; 33(2): 555-60. 
23. Massry SG. Is parathyroid hormone a uremic toxin? Nephron. 1977; 19(3):125-30.

24. Liang K, Oveisi F, Vaziri ND. Role hyperparathyroidism in the genesis of hypertriglyceridemia and VLDL receptor deficiency in chronic renal failure. Kidney Int. 1998; 53(3):620-30.

25. Vaziri ND, Wang XQ, Liang K. Secondary hyperparathyroidism dowregulates lipoprotein lipase expression in chronic renal failure. Am J Physiol. 1997; 273(6 Pt2):F925-30.

26. Nishizawa $Y$, Shoji T, Kawagishi T, Morii H. Atherosclerosis in uremia: possible roles of hyperparathyroidism and intermediate density lipoprotein accumulation. Kidney Int. 1997; 62:590-2.

27. Tzanno-Martins C, Futata E, Jorgetti V, Duarte AJ. Immune response in hemodialysis patients: Is there any difference when low and hight iPTH levels are compared? Clin Nephrol. 2000; 54(1):22-9.

28. Baczynski R, Massry SG, Magott M, el-Belbessi S, Kohan R, Brautbar N. Effect of parathyroid hormone on energy metabolism of skeletal muscle. Kidney Int. 1985; 28(5):722-7.

29. Hsu CH. Historical perspective on management of calcium and phosphorus metabolism in chronic renal failure. Am J Kidney Diseases. 2001; 37(1):195-201.

30. Dumler F, Kilates $C$. Use of bioelectrical impedance techniques for monitoring nutritional status in patients on maintenance dialysis. J Renal Nutr. 2000; 10(3):116-24.

31. Monteiro JBR, Silva RR, Rosado EL, Gouveia MG, Norfino SP. Uso de bioimpedância elétrica e pregas cutâneas em pacientes submetidos a hemodiálise. Rev Bras Nutr Clin. 1999; 14:220-7.
32. Kamimura MA, Santos NSJ, Avesani CM, Fernandes Canziani ME, Draibe SA, Cuppari L. Comparison of three methods for the determination of body fat in patients on long-term hemodialysis therapy. J Am Dietetic Ass. 2003; 103(2):195-9.

33. Kamimura MA, Avesani CM, Cendoroglo $M$, Canziani ME, Draibe SA, Cuppari L. Comparison of skinfold thicknesses and bioelectrical impedance analysis with dual-energy X-ray absorptiometry for the assessment of body fat in patients on long-term hemodialysis therapy. Nephrol Dial Transplant. 2003; 18(1):101-5.

34. Mucsi I, Hercz G, Uldall R, Ouwendyk M, Francouer $R$, Pierratos $A$. Control of serum phosphate whithout any phosphate binders in patients treated with nocturnal hemodialysis. Kidney Int. 1998; 53(5):1399-404.

35. Silver J, Kilav R, Naveh-Many T. Mechanisms of secondary hyperparathyroidsm. Am J Physiol Renal Physiol. 2002; 283(3):F367-76.

36. Calvo, MS. Dietary considerations to prevent loss of bone and renal function. Nutrition. 2000; 16(7-8):564-6

37. Avram MM, Mittman N, Myint MM, Fein P. Importance of low serum intact patathyroid hormone as a predictor of mortality in hemodialysis and peritoneal dialysis patients: 14 years of prosective observation. Am J Kidney Dis. 2001; 38(6):1351-7.

38. Cupisti A, Morelli E, D'Alessandro C, Lupetti S, Barsotti G. Phosphate control in chronic uremia: Don't forget diet. J Neprol. 2003; 16(1):29-33.

Recebido em: 10/5/2004

Versão final reapresentada em: 7/4/2005 Aprovado em: 28/4/2005 